Georgia State University

ScholarWorks @ Georgia State University

$1-1-2006$

\title{
Direct Tests of Models of Social Preferences and a New Model
}

James Cox

Georgia State University

Vjollca Sadiraj

Georgia State University

Follow this and additional works at: https://scholarworks.gsu.edu/excen_workingpapers

\section{Recommended Citation}

Cox, James and Sadiraj, Vjollca, "Direct Tests of Models of Social Preferences and a New Model" (2006). ExCEN Working Papers. 136.

https://scholarworks.gsu.edu/excen_workingpapers/136

This Article is brought to you for free and open access by the Experimental Economics Center at ScholarWorks @ Georgia State University. It has been accepted for inclusion in ExCEN Working Papers by an authorized administrator of ScholarWorks @ Georgia State University. For more information, please contact scholarworks@gsu.edu. 


\title{
Direct Tests of Models of Social Preferences and a New Model
}

\author{
By James C. Cox and Vjollca Sadiraj
}

\begin{abstract}
Departures from "economic man" behavior in many games in which fairness is a salient characteristic are now well documented in the experimental economics literature. These data have inspired development of new models of social preferences incorporating inequality aversion and quasi-maximin preferences. We report experiments that provide direct tests of these social preference models. Data from the experiments motivate a new model of egocentric altruism. The model rationalizes data from our direct test experiments and data from experiments with proposer competition and responder competition. We discuss generalizations of the egocentric altruism model that incorporate agents' intentions and thus provide a unified approach to modeling behavior in games both with and without reciprocal motivation.
\end{abstract}

Keywords: social preferences, fairness, experiments

JEL Classifications: A12, A13, B49, C70, C91, D63 


\section{Introduction}

Economics has a long history of using models of preferences. The preferences are conventionally represented by utility functions and their indifference maps. Until recently, the preferences most commonly used have been self-regarding (or "economic man”) preferences in which an agent cares about his own material payoffs but is indifferent about the material payoffs of others. There is now a large literature that supports the conclusion that self-regarding preference models are mostly inconsistent with behavior in experiments in which distributional fairness is a salient characteristic. This has motivated the development of new models of other-regarding (or "social”) preferences.

We report direct tests of the central properties of recent prominent contributions to modeling social preferences, including inequality aversion models (Fehr and Schmidt, 1999 and Bolton and Ockenfels, 2000) and a quasi-maximin model (Charness and Rabin, 2002). The distinguishing characteristic of inequality aversion models is that utility is decreasing with the difference between one's own and others' material payoffs. The distinguishing characteristic of the quasi-maximin model is that utility is increasing with the lowest of all agents' payoffs (the maximin property) and the total of all agents' payoffs (the efficiency property). These distinguishing characteristics of the two types of models provide the basis for our direct tests of the models.

Fairness game data come from experiments with different types of games, including: (a) reciprocal-motivation games, such as the ultimatum game, in which beliefs about others' possible future actions and imputations of the intentions behind their past actions can affect agents' behavior; and (b) simple distribution games, such as the dictator game, in which such beliefs and imputations are irrelevant (within the context of the experiment). The inequality aversion and quasi-maximin models that we test are models of preferences that are not conditional on others' revealed intentions nor beliefs about their future actions. Therefore, it is appropriate to conduct direct tests of these models with experiments involving simple distribution games rather than 
reciprocal-motivation games. Our tests are conducted with specially-designed dictator games that are constructed to test the central distinguishing characteristics of the models.

Data from the experiments are mostly inconsistent with the inequality aversion and quasimaximin models. Furthermore, the data do not indicate noisy or random behavior by the subjects in the experiments. Instead, the data are mostly consistent with a model of other-regarding preferences with the conventional regularity properties of positive monotonicity and strict convexity (of indifference curves). These data motivate our development of a model of egocentric altruism in which preferences are positively monotonic and strictly convex. This model is applied to data from our four experiments and data from experiments with proposer competition (Roth, et al., 1991) and responder competition (Güth, Marchand, and Rulliere, 1997).

Our strategy in modeling other-regarding preferences is to implement a unified approach that develops a model that is consistent with behavior in simple distribution games and then generalizes that model to incorporate intentions. This paper focuses on modeling behavior in simple distribution games. We also explain how the model developed here can be extended in a straightforward way to incorporate intentions. This modeling strategy provides a unified approach to modeling behavior in games both with and without reciprocal motivation.

\section{Experiment 1: A Direct Test of Inequality Aversion}

Inequality aversion models are based on the assumption that an agent's utility is increasing with her own material payoff but decreasing with the difference between her own and others' material payoffs. For the special case of two agents and (scalar) money payoffs, the fundamental property of inequality aversion models is that the indifference curves have positive slopes in the part of the money payoff space in which the other's payoff is higher than one's own. This property forms the basis of a direct test for inequality aversion. 


\subsection{Experimental Design and Procedures}

Experiment 1 involves a dictator game with the following characteristics. Subjects are randomly assigned to pairs. In addition to a show-up fee of $\$ 5$, each subject in a pair is given an endowment of $\$ 10$. The "non-dictators" have no decision to make. The dictators are told that they can send zero or a positive amount (in whole dollar units), up to $\$ 10$, from their endowment to the other person. Each dollar that a dictator transfers to the other person is multiplied by three by the experimenters. The experimental protocol uses double-blind payoff procedures in which neither the other subjects nor the experimenters can identify the individual who has chosen any specific action. All of the features of the experiment, including the equal endowments of dictators and non-dictators, are common information given to the subjects. The experiment procedures are described in detail in Appendix 1. The subject instructions are available on request to the authors.

\subsection{Predictions of the Inequality-Aversion Models}

Figure 1 shows typical indifference "curves" for the Fehr and Schmidt (1999) model for the dictator's (“my”) money payoff $m$ and the other subject’s (“your”) money payoff $y$. All parameter values for this model that are consistent with its defining characteristic of inequality (or “inequity”) aversion imply that the indifference "curves” have positive slope above the 45-degree line. (See Appendix 2 for derivation of these properties of the indifference "curves.”) Including the $\$ 5$ show-up fees in payoffs, the budget constraint of a dictator in experiment 1 consists of ordered pairs of integers on the dashed line in Figure 1 extending from the point $(15,15)$ on the 45-degree line to the point $(5,45)$ near the vertical axis. In this dictator game, the Fehr-Schmidt model predicts that a dictator will give 0 dollars to the other subject.

Figure 2 shows typical graphs of the level sets or indifference curves of the Bolton and Ockenfels (2000) "motivation function" for the two-agent case with $m+y>0$. (See Appendix 2 for derivation of these properties of the indifference curves.) This model predicts that the dictator 
will give 0 dollars to the other subject for the same reason as does the Fehr-Schmidt model: above the 45-degree line, the indifference curves have positive slope whereas the budget line has negative slope.

\subsection{Subjects’ Behavior in Experiment 1}

Data from experiment 1 are reported in Figure 3 with the light-colored bars. In this experiment 19 of 30 or $63 \%$ of the dictators gave positive amounts to the other person and, hence, exhibited behavior that is inconsistent with inequality aversion. The $63 \%$ of dictators who sent positive amounts of money to the other subjects imposed significant costs on themselves to increase inequality favoring others. This behavior is inconsistent with the central distinguishing characteristic of inequality aversion models. The average amount given away by the dictators was $\$ 3.60$, which gave the average recipient a payoff of $\$ 25.80(=\$ 5+\$ 10+3 \times \$ 3.60)$ and left the dictators with an average payoff of $\$ 11.40$ (= $\$ 5+\$ 10$ - \$3.60). Furthermore, the behavior of the $37 \%$ of subjects who did not give any money to the paired subject can be explained by selfregarding (or “economic man”) preferences. Therefore, inequality aversion is not needed to explain the behavior of any subject in this experiment.

\subsection{Related Experiments}

Comparison of data from experiment 1 with data from other dictator experiments provides additional insight into the properties of subjects' preferences. In the (DB1 and DB2) double-blind dictator experiments reported by Hoffman, McCabe, Shachat, and Smith (1994), the average amount sent to the paired subjects by the dictators was $\$ 1$. In our experiment 1 dictator game, the average amount sent by the dictators was $\$ 3.60$. The price to the dictator of buying an additional $\$ 1$ of income for the paired subject was $\$ 1$ in the Hoffman, et al. experiment and it is $\$ 0.33$ in our 
experiment 1 . The implied (arc) price elasticity of demand for increasing the other subject's income is -1.13 , a quite reasonable figure.

Other papers that report experimental tests of inequality aversion models (Charness and Rabin, 2002; Deck, 2001; and Engelmann and Strobel, 2004) also find that the models' predictions are inconsistent with the behavior of a large proportion of the subjects. Therefore there is a moderately large body of data that are inconsistent with inequality aversion, which suggests the need for a different type of model. After reporting their tests of inequality aversion, Charness and Rabin introduce the quasi-maximin model and apply it to data from several experiments. We next report direct tests of that model.

\section{Direct Tests of Quasi-Maximin Preferences}

\subsection{The Quasi-Maximin Model}

Let $x$ denote a vector of money payoffs of $n$ agents and $x_{i}$ denote the payoff of agent $i$. Charness and Rabin’s (2002) “reciprocity-free” model is based on the assumption that the utility function of agent $i$ is increasing with the amount of her own money payoff $\left(x_{i}\right)$, the minimum of all agents' payoffs $\left(\min _{j \in\{1, \ldots, n\}}\left\{x_{j}\right\}\right)$, and the total of all agents' payoffs $\left(\sum_{j=1}^{n} x_{j}\right)$. The quasi-maximin utility function is assumed to be:

$$
u^{i}(x)=(1-\gamma) x_{i}+\gamma\left[\delta \min _{j \in\{1, \ldots, n\}}\left\{x_{j}\right\}+(1-\delta) \sum_{j=1}^{n} x_{j}\right],
$$

where $\gamma \in[0,1]$ and $\delta \in(0,1)$. The $\gamma$ parameter measures the relative importance of own money payoff compared to the two other arguments of the utility function. The $\delta$ parameter measures the relative importance of these other two arguments, the minimum payoff and total payoff (or “efficiency”). 


\subsection{Experiments 2 and 3: Tests of Quasi-Maximin Preferences}

The three arguments of utility function (1) suggest the design of two experiments that provide direct tests for quasi-maximin preferences. In experiment 2, we offer subjects choices between alternatives in a dictator game in which the dictator's own payoff and the minimum payoff are constant but the sum of all payoffs changes. The three rows in Table 1 show the choices open to a subject in experiment 2 when the $\$ 5$ show-up fees are included in payoffs. Because the dictator's payoff is the same in all rows and the lowest payoff is the same in all rows, the quasi-maximin model predicts that an agent will choose row 3, which has the highest total payoff to all agents (except in the limiting case in which $\gamma=0$, where the model makes no prediction because this is the special case of self-regarding preferences). This row 3 prediction is independent of the piecewise linear form of the Charness-Rabin (2002) utility function. Thus the experiment provides a direct test of the assumed preference for efficiency per se.

Whereas experiment 2 tests for a preference for efficiency, experiment 3 tests for the other defining property of the quasi-maximin model, the preference for increasing the payoff to the lowest paid agent (the maximin property). Thus, in experiment 3, we offer subjects choices in a dictator game in which the dictator's own payoff and the total payoff are constant but the minimum payoff changes. The three rows in Table 2 show the choices open to a subject in this dictator experiment when the \$5 show-up fees are included in payoffs. Since the dictator's payoff is the same in all rows and the total payoff to all agents is the same in all rows, the quasi-maximin model predicts that an agent will choose row 3, which has the maximin payoff (except in the limiting case in which $\gamma=0$, where the model makes no prediction because this is the special case of self-regarding preferences). This row 3 prediction is independent of the piece-wise linear form of the Charness-Rabin (2002) utility function. Thus the experiment provides a direct test of the assumed maximin property per se.

\subsection{Procedures in Experiments 2 and 3}


Experiments 2 and 3 have the following characteristics. Subjects are randomly assigned to groups of four that consist of a dictator and three "non-dictators." The dictators are told that they must choose one row from Table 1 in experiment 2 or one row from Table 2 in experiment 3 . Different subjects participated in experiments 2 and 3 . The experimental protocol uses double-blind payoff procedures in which neither the other subjects nor the experimenters can identify the individual who has chosen any specific action. All of the features of the experiment are common information given to the subjects. The experiment procedures are described in detail in Appendix 1. The subject instructions are available on request to the authors.

\subsection{Behavior in Experiments 2 and 3}

Subjects' behavior in experiments 2 and 3 is reported in Figure 4. We observe that only 5 of 33 (or 15\%) of the subjects chose row 3 in experiment 2, which is the unique prediction of the quasimaximin model. Thus, the behavior of $85 \%$ of the subjects in experiment 2 is inconsistent with quasi-maximin preferences. In experiment 3, only 2 of 32 (or 6\%) of the subjects chose row 3, which is the unique prediction of the quasi-maximin model. Hence the behavior of $94 \%$ of the subjects in experiment 3 is inconsistent with the quasi-maximin model.

\section{The Egocentric Altruism Model}

The very high rates of inconsistency between subjects' behavior and the testable implications of the inequality-aversion and quasi-maximin models suggest the need for a model with different properties. As it turns out, the behavior observed in experiments 1-3 can be rationalized by a utility function with the conventional properties of strict quasi-concavity (i.e., indifference curves that are strictly convex to the origin) and positive monotonicity (i.e., increasing) in the dictator's income $x_{i}$ and others' incomes $x_{j}, j \in\{1, \ldots, n\} \backslash\{i\}$. 
Andreoni and Miller (2002) test data from many dictator games, with varying budgets and own-payoff prices for altruistic actions, for consistency with utility-maximizing behavior by testing the data for consistency with the generalized axiom of revealed preference (GARP). They report that 98 percent of their subjects make decisions that are consistent with GARP and therefore are, in that specific sense, rational altruists. Furthermore, Andreoni and Miller report that constant elasticity of substitution (CES) parametric utility functions provide a good fit to data for their subjects. We develop a model based on CES utility functions that are modified to capture salient characteristics of subjects' altruistic preferences that are revealed by our experiments. We begin by presenting the two-agent special case of the model.

\subsection{The Two-Agent Egocentric Altruism Model}

The two-agent special case of the egocentric altruism model represents other-regarding preferences with a modified CES utility function of an agent’s own (“my”) money payoff $m$ and the other agent's ("your") money payoff $y$ that is weighted by the altruism parameter $\theta \geq 0$ :

$$
\begin{aligned}
u(m, y) & =\frac{1}{\alpha}\left(m^{\alpha}+\theta y^{\alpha}\right), & & \alpha \in(-\infty, 1) \backslash\{0\}, \\
& =m y^{\theta}, & & \alpha=0 .
\end{aligned}
$$

This utility function is assumed to be monotonically increasing in $m$ and $y$ and to have indifference curves that are negatively-sloped and strictly convex to the origin except for the boundary value of $\theta=0$, in which case the model is equivalent to the model of self-regarding preferences. For $\theta>0$, the special case of $\alpha=0$ is the Cobb-Douglas utility function given in the second line of statement (2).

The agent's altruistic preferences are assumed to be "egocentric," by which we mean that between two money allocations $(a, b)$ and $(b, a)$ the agent prefers the one that allocates the larger payoff to himself:

(3) $\quad u(b, a)>u(a, b)$, for all $a$ and $b$ such that $b>a \geq 0$. 
Together, the assumptions of monotonicity, convexity, and egocentricity imply the following restrictions on the parameters of the utility function:

$$
\alpha<1 \text { and } \theta \in[0,1) \text {. }
$$

Differentiation of (2) yields the marginal rate of substitution between $y$ and $m$ :

$$
\operatorname{MRS}(m, y)=\frac{1}{\theta}\left(\frac{y}{m}\right)^{1-\alpha}
$$

Statements (4) and (5) imply that $M R S(m, y)$ : (a) is everywhere positive (monotonicity); (b) does not vary with $m$ and $y$ when relative income, $y / m$, is held constant (homotheticity); (c) increases with $y / m$ (indifference curve convexity to the origin); and (d) is greater than 1 when $y / m=1$ (egocentricity).

\subsection{The Many-Agent Egocentric-Altruism Model}

The generalization of the egocentric altruism utility function to $n \geq 2$ agents is:

$$
\begin{array}{rlrl}
u^{i}(x) & =\frac{1}{\alpha}\left[x_{i}^{\alpha}+\theta \sum_{j \neq i} x_{j}^{\alpha}\right], & \alpha \in(-\infty, 1) \backslash\{0\}, \\
& =x_{i}\left(\prod x_{j}\right)^{\theta}, & & \alpha=0 .
\end{array}
$$

where $x_{i}$ is the money payoff of agent $i$ and $x_{j}, j \neq i$ is the money payoff of agent $j$. The egocentric property generalizes to the $n$-agent case as follows. First define:

$$
\begin{aligned}
& x^{a, b}, x^{b, a} \in \mathfrak{R}_{+}^{n} \text { such that } x_{i}^{a, b}=x_{k}^{b, a}=a, x_{k}^{a, b}=x_{i}^{b, a}=b \text {, for some } i \text { and } k \text {, and } \\
& x_{j}^{a, b}=x_{j}^{b, a} \text { for all } j \in\{1, \ldots, n\} \backslash\{i, k\} .
\end{aligned}
$$

Then the egocentricity property is

$$
u^{i}\left(x^{b, a}\right)>u^{i}\left(x^{a, b}\right) \text { for all } x^{a, b}, x^{b, a} \text { and } a, b \text { such that } b>a \geq 0 \text {. }
$$

The parameter restrictions implied by monotonicity, egocentricity, and strict convexity are given by statement (4). 


\section{Explanatory Power of the Egocentric Altruism Model}

We first show that the egocentric altruism model is consistent with behavior in dictator game experiments 1, 2 and 3. We next report another dictator game experiment (experiment 4) that reveals other properties of subjects' preferences that can be rationalized by the model. Lastly, we show that the model can rationalize data from experiments with proposer competition (Roth, et al., 1991) and responder competition (Güth, Marchand, and Rulliere, 1997). This procedure provides a check on how robust the model is to explaining behavior in distribution games.

\subsection{Is the Egocentric Altruism Model Consistent with Behavior in Experiments 1-3?}

We first ask whether the behavior of subjects in experiment 1 is consistent with the egocentric altruism model. In other words, are the data from experiment 1 consistent with the two-agent utility function given by equation (2) and the parameter restrictions in statement (4) that are implied by monotonicity, egocentricity, and convexity?

In experiment 1, a dictator can choose a whole dollar amount $s$ weakly between 0 and 10 to send to the paired subject. Each $\$ 1$ sent decreases the dictator's money payoff by $\$ 1$ and increases the paired subject’s money payoff by $\$ 3$. Hence, the slope of the dictator's budget line is -3 above the 45 -degree line. Therefore, if the slope of an indifference curve ( $-M R S$ ) at the point $(15-1,15+3)$, which corresponds to giving one dollar to the other person, is the same or larger than the slope of the budget line $(-3)$ then the most preferred option for the dictator is to give at least one dollar to the other person. If this is not true then the dictator's most preferred choice is to give 1 or 0 , depending on which of these two (whole dollar amounts) has larger utility. Appendix 3 derives this formally and shows that there exists a function of $\alpha$, $v:(-\infty, 1) \rightarrow[1 / 3,1)$ such that a dictator with $\theta / v(\alpha)>1$ will give at least $\$ 1$ of her endowment

to the paired subject whereas a dictator with $\theta / v(\alpha)<1$ will send 0 . (For $\theta=v(\alpha)$ the dictator is indifferent between giving $\$ 1$ or $\$ 0$ ). 
The data reported in Figure 3 reveal that $63 \%$ of the subjects made choices in experiment 1 consistent with $\theta / v(\alpha)>1$ and $37 \%$ made the choice consistent with $\theta / v(\alpha)<1$. Specific $(\alpha, \theta)$ parameter values can rationalize each of the distinct observations in experiment 1.

We now ask whether the subjects' behavior in experiments 2 and 3 is consistent with the egocentric altruism model. In other words, are the data from experiments 2 and 3 consistent with the many-agent utility function given by equation (6) and the parameter restrictions in statement (5) that are implied by monotonicity, egocentricity, and convexity?

With respect to experiment 2, the egocentric altruism model with $\theta \neq 0$ ranks row 2 higher than row 1 because the utility function is positively monotonic in all payoffs and some row 2 payoffs are larger than the corresponding row 1 payoffs and no row 2 payoffs are lower than corresponding row 1 payoffs. With respect to rows 2 and 3, a dictator with $\alpha=0$ always prefers row 2 to row 3 because $15(5 \times 20 \times 20)^{\theta}>15(5 \times 7 \times 38)^{\theta}$. A dictator with $\alpha \neq 0$ prefers row 2 to row 3 if $\alpha<.593$ and she prefers row 3 to row 2 if $\alpha>.594$. In the later case, the most preferred row is row 3 because, from transitivity, if row 2 is preferred to row 1 and row 3 is preferred to row 2 then row 3 is preferred to row 1 . We observe from Figure 4 that the egocentric altruism model is consistent with the behavior of 28 out of 33 (or 85\%) of the subjects in experiment 2 who chose either row 2 or row 3 . The $70 \%$ of dictators who chose row 2 reveal $\alpha<.593$ and the $15 \%$ of the subjects who chose row 3 reveal $\alpha>.594$.

In experiment 3, row 2 is preferred to row 1 since the utility gain $\left(8^{\alpha}-5^{\alpha}\right) \theta / \alpha$ from an increase of the payoff of agent 1 by 3 units is always larger than the decrease in the utility, $\left(20^{\alpha}-17^{\alpha}\right) \theta / \alpha$, from a reduction of the payoff of agent 2 by 3 units. If $\alpha=0$ then one has $15(8 \times 17 \times 20)^{\theta}>15(3 \times 20 \times 20)^{\theta}$, and therefore again row 2 is preferred to row 1 by such a dictator. Similarly, it can be verified that row 2 is preferred to row 3 as well, and therefore the egocentric altruism model predicts that the dictator will choose row 2 in experiment 3 . We 
observe from Figure 4 that 28 of 32 subjects chose row 2 in experiment 3; hence the egocentric altruism model is consistent with the behavior of $88 \%$ of the subjects in that experiment.

\subsection{Experiment 4: How Do Dictators Respond to the Opportunity to Take Money?}

A common feature of experiments $1-3$ is that the dictator cannot take money from another subject and appropriate it himself. This means that the designs of those experiments do not cause the subjects to reveal information about some characteristics of their preferences. In order to construct an experiment that will reveal more about the subjects' preferences, we expand the feasible set of experiment 1 to include opportunities to take money as well as give it away.

\subsubsection{Experimental Design and Procedures}

In experiment 4, subjects are randomly assigned to pairs. Each subject in a pair is given $\$ 10$ plus a show-up fee of $\$ 5$. The "non-dictators" have no decision to make. The dictator is asked to decide whether he wants to give up to $\$ 10$ from his own endowment to the other subject or take up to $\$ 5$ of the other subject's endowment or neither give nor take anything. Amounts given or taken have to be in whole dollar amounts. Any amount given to the other subject is multiplied by three by the experimenter. Each dollar taken from the other subject increases the dictator's payoff by one dollar; that is, there is no multiplication by three by the experimenter. The experimental protocol uses double-blind payoff procedures. All of the features of the experiment, including the equal endowments, are common information given to the subjects. In summary, experiment 4 differs from experiment 1 only by introduction of the opportunity to take money from the paired subject. The experiment procedures are described in Appendix 1. The subject instructions are available on request to the authors. 


\subsubsection{Subjects' Behavior in Experiment 4}

Data from experiment 4 are reported in Figure 3 with the dark-colored bars. Note that 22 of 32 or $69 \%$ of the dictators took money from the other person and 18 of 32 or $56 \%$ took the maximum possible amount, $\$ 5$. Also, 3 of 32 or $9 \%$ of the dictators gave money to the other person and 7 of 32 or $22 \%$ neither gave nor took any money (they chose $\$ 0$ as the amount to send or take). Note that $22 \%$ of the dictators chose equal payoffs and $78 \%$ chose unequal payoffs.

Figure 3 shows very different outcomes in experiments 1 and 4 . Introduction of the opportunity to take money changes the distribution of behavior from appearing to be predominantly altruistic to appearing to be predominantly selfish. Just such behavior is consistent with a model with down-sloping indifference curves that are strictly convex to the origin. For example, Figure 5 shows indifference curves (for $\alpha=0.5$ and $\theta=0.5$ ) that would lead the same agent to give \$4 in experiment 1 and take \$5 in experiment 4.

Although Figure 5 shows how the egocentric altruism model is consistent with giving in experiment 1 and taking in experiment 4, alternative parameter values are consistent with the behavior of subjects who give money or choose $s=0$ in experiment 4 . For example, if $\alpha=0.5$ and $\theta=0.6$ then the optimal choice is to give $\$ 5$ in both experiments 1 and 4 . If $\alpha=-6$ and $\theta=0.7$ then the optimal choice in both experiments 1 and 4 is $s=0$.

The implications of the model for behavior in experiment 4 are as follows. The dictator's budget line in this experiment is piecewise linear, with slope that increases from -3 above the 45-degree line to -1 below it. Indifference curves of utility function (2) have negative slopes that decrease as $y / m$ increases. Hence, if the slope at the payoff vector $(m, y)=(16,14)$, implied by taking $\$ 1$, is smaller than -3 (which is smaller than -1 ) then so are the slopes at all points with higher $y / m$. This implies that the optimal choice is to take at least $\$ 1$. On the other hand if the slope at the payoff vector resulting from giving $\$ 1$ is larger than -1 (which is larger than -3 ) then so is the slope at all points with lower $y / \mathrm{m}$. Hence, in that case, the best choice is to give at 
least \$1. If however, the slope of an indifference curve at the payoff vector resulting from taking $\$ 1$ is larger than -1 , and the slope from giving $\$ 1$ is smaller than -3 , then the dictator's optimal choice is to take $\$ 1$, give $\$ 1$, or choose $\$ 0$ depending on which of them has the highest utility. Appendix 3 presents a complete derivation of the model's predictions for experiment 4 . It is shown there that there exist three functions of the $\alpha$ curvature parameter, $h, v$, and $w$ such that a dictator with $\theta<h(\alpha)$ will take money if $\theta<w(\alpha)$ or choose 0 if $\theta>w(\alpha)$, whereas a dictator with $\theta>h(\alpha)$ will either give a positive amount of money if $\theta>v(\alpha)$ or choose 0 if $\theta<v(\alpha)$

\section{$\underline{5.3 \text { Explaining Competition }}$}

We next consider the question of how robust is the empirical consistency of the egocentric altruism model with behavior. We here consider experiments that involve competition among the agents on "one side" of the game.

\subsubsection{Game with Proposer Competition}

Roth, Prasnikar, Okuno-Fujiwara, and Zamir (1991) report results from experiments in four countries with the following game of proposer competition. $n-1$ proposers can simultaneously propose $s_{j} \in[0, S], j=1,2, \cdots, n-1$ in discrete increments. The responder can accept or reject the highest offer, $\bar{s}=\max \left\{s_{1}, s_{2}, \cdots s_{n-1}\right\}$. If the responder accepts the highest offer then the proposer who made the offer gets $S-\bar{s}$, the other proposers get 0 , and the responder gets $\bar{S}$. If more then one proposer makes the accepted offer then one of the proposers is randomly selected, with equal probability for all tied proposers, to get $S-\bar{S}$. The experiments included several response periods. One period was randomly selected for money payoff to the subjects. The predictions of the egocentric altruism model for this experiment are as follows. 
A formal proof is provided in Appendix 3. Here we offer an informal demonstration that conveys intuition about the implications of the egocentric altruism model for subgame perfect equilibrium in the game with proposer competition. The responder prefers the money payoffs from accepting any $\bar{s} \geq 0$ to payoffs from rejecting it because, in the former case, at least one player has a strictly higher payoff and utility function (6) is monotonically increasing in all payoffs. Thus the responder will accept all offers. Because of egocentricity and convexity, a proposer prefers the payoffs from the set of offers in which he is one of $k$ proposers, $1<k \leq n-1$, who submits $\bar{s}>0$ and has $1 / k$ probability of receiving $S-\bar{s}$, to the payoffs from $s^{P}<\bar{s}$ where he gets 0 for sure (in addition to the show-up fee). Thus one has the following proposition.

Proposition 1. Let $\bar{s}$ be the highest offer submitted by proposers in the game of proposer competition. Then

1) The responder will accept all offers;

2) For proposers:

a) all proposers offering $S-S_{*}$ is a subgame perfect symmetric equilibrium;

b) any vector of offers with at least two proposers offering $S$ is a subgame perfect asymmetric equilibrium;

c) there are no asymmetric subgame perfect equilibria with the highest offer less than S.

In the Roth, et al. (1991) study, $S=1000$ and $S_{*}=5$. Data from their experiment show that 72 (resp. 14) out of 158 proposers offered 995 (resp. 1000) at round 10. In that round 2 out of 18 groups are settled in the equilibrium given in part 2.b of Proposition 1. If we allow for an error of two players, that is if at least 7 (resp. 5) out of 9 (resp. 7) offer $995\left(=S-S_{*}\right)$, then 6 out of 18 groups are settled at the symmetric equilibrium given part by 2.a of Proposition 1. 


\subsubsection{Game with Responder Competition}

Güth, Marchand, and Rulliere (1997) report an experiment with a game in which a proposer makes an offer $s, s \in[0, S]$ to $n-1$ responders. In this experiment $S$ is 50 French francs and $n=6$. A responder can accept or reject the proposal. If only one of the responders accepts the offer then she gets $S$, the other responders get 0 , and the proposer gets $S-s$. If more then one responder accepts the proposal then one of the responders is randomly selected to get $s$. If all of the responders reject the proposal then all players get 0 . The experiments were run with a design in which responders were asked to pre-commit to acceptance thresholds for a period before observing the proposal for that period. The experiment included several response periods. Subjects received money payoffs for every period. Subjects were informed at the end of each period of their own payoff but not the payoffs received by others. The predictions of the egocentric altruism model for this experiment are as follows.

Appendix 3 presents a formal proof of the egocentric altruism model's subgame perfect equilibrium for the game with responder competition. Here we present an informal demonstration that conveys intuition about the properties of the equilibrium. Consider any proposal, $s \geq 0$. If all responders reject the proposal, everyone gets money payoff of 0 . Therefore, a responder prefers to accept any $s \geq 0$, rather than accept the outcome where everyone gets 0 , because of the positive monotonicity of utility function (6). If $k$ other responders, $1 \leq k \leq n-2$, accept $s>0$, the utility function implies that a responder also prefers to accept $s>0$ because the payoffs determined by a $1 /(k+1)$ probability of receiving $s>0$ are preferable to those where the responder receives 0 for sure. Since responders will accept any proposal, the proposer will propose the offer $s^{P}$ that maximizes his own utility function. One responder will be randomly selected to receive $s^{P}$ and the proposer will receive $\left(S-s^{P}\right)$. Let the vector of payoffs be $x=\left(x_{j}\right)_{j=1, \ldots, n}$ with the first element the proposer's payoff. In the experiment, the proposer does 
not know which responder received the payoff determined by the proposal in any preceding round. Since the responders' best reply is to accept everything, it is reasonable to assume that the proposer believes that the responders are equally likely to receive the proposed payoff in any round of the experiment. Thus the proposer's expected payoff for each responder $j$ after round $t$ is $x_{j}=E\left(x_{j, t}\right)=x_{0}+\frac{1}{n-1} \sum_{r=1 . . t} s_{r}^{P}(\equiv y), j=2, \ldots, n$, where $x_{0} \geq 0$ is the show-up fee. The payoff of the proposer is $x_{1}=x_{0}+\sum_{r=1, t}\left(S-s_{r}^{P}\right)$. Hence, the proposer's expected utility is $\left(^{*}\right)$ $E U(s)=\frac{1}{n-1} \sum_{k \neq i} U_{k}(s)$, where

$$
\begin{aligned}
U_{k}(s) & =\frac{1}{\alpha}\left[\left(x_{1}+S-s\right)^{\alpha}+\theta\left(\left(x_{k}+s\right)^{\alpha}+(n-2) y^{\alpha}\right)\right] \quad \alpha \in(-\infty, 1] \backslash\{0\}, \\
& =\left(x_{1}+S-s\right)\left(\left(x_{k}+s\right) y^{n-2}\right)^{\theta} \quad \alpha=0 .
\end{aligned}
$$

Substituting (9) in $(*)$ one has

$$
\begin{gathered}
E U(s)=\frac{1}{\alpha}\left[\left(x_{1}+S-s\right)^{\alpha}+\theta\left((y+s)^{\alpha}+(n-2) y^{\alpha}\right)\right], \quad \alpha \in(-\infty, 1] \backslash\{0\}, \\
=\left(x_{1}+S-s\right)\left((y+s) y^{n-2}\right)^{\theta}, \quad \alpha=0 .
\end{gathered}
$$

This is the intuition behind the following proposition that is proved in Appendix 3.

Proposition 2. In the subgame perfect equilibrium:

1) All responders accept all proposals;

2) The proposer's offer $s^{P}$ maximizes expected utility function (10).

Data from the experiment show that the percentage of responders choosing 0 thresholds to be $13 \%, 30 \%, 40 \%, 50 \%$ and $67 \%$ in the first to fifth rounds. Although the responders' behavior has not fully converged by round 5 (the last round), continuation of this monotonic convergence pattern to at least 8 rounds would have had $100 \%$ of responders choosing 0 thresholds. Thus the monotonic convergence pattern in the data appears to be consistent with part 
1 of Proposition 2. Proposers' behavior does appear to have converged by round 4 because, from the third round on, there is always at least one responder with a 0 (resp. 1) threshold in 10 (resp. 12) out of 12 groups. Therefore, the proposers can be assured that their proposals will be accepted by at least one responder and, therefore, they can choose proposals that maximixe their utility. The data show that 11 (resp. 8) out of 12 proposers offer at least 5 (resp. 10) French francs, which reveals that proposers have altruistic preferences, because proposers with self-regarding preferences would choose 0 when assured of acceptance by at least one responder.

\section{Concluding Remarks}

As shown in Charness and Rabin (2002), Deck (2001), Engelman and Strobel (2004), and section 2 above, a large majority of subjects make choices that are inconsistent with inequality aversion models in dictator game experiments designed to provide direct tests for inequality aversion. As shown in section 3, most subjects make choices that are inconsistent with the quasi-maximin model in dictator game experiments that are designed to directly test the central defining characteristics of the model. In contrast, the egocentric altruism model is consistent with the behavior of most of the subjects in all four different types of dictator games. Furthermore, the egocentric altruism model is consistent with the behavior of a high proportion of subjects in competitive experiments reported in the literature, including experiments with proposer competition and responder competition. Thus the empirical success of the egocentric altruism model is robust to several distinct types of experiments run by different researchers. The common feature of these experiments is that they involve games that do not elicit reciprocal motives; they are dictator games or games in one-sided competitive environments. This suggests the important question of whether the egocentric altruism model can be generalized to incorporate reciprocal motives, thus holding out the promise of an empirically-motivated and unified approach to modeling social preferences in environments both with and without reciprocal motives. 
Two generalizations of the two-agent egocentric altruism model are being developed, one parametric and the other non-parametric. In the parametric model, the weight on the other person's payoff in the agent's CES utility function depends on the kindness or unkindness of others' choices (their revealed intentions) and on their status relative to the agent. The parametric model incorporating intentions and status is applied to data from several types of games with reciprocal motivations and with and without induced status differences. In the nonparametric model, the indifference curves of the utility function are always convex to the origin, but the marginal rate of substitution between ones' own and another's payoff depends on the other's previous actions. Two partial orderings are introduced, an ordering of preferences by a formal representation of "more altruistic than" and an ordering of opportunity sets by a formal representation of "more generous than.” These partial orderings are linked by: (a) the "reciprocity axiom” which specifies that more generous choices by a first mover induce more altruistic preferences in a second mover; and (b) the "status quo axiom" which specifies that generous acts of commission induce more altruistic preferences than equally-generous acts of omission. The nonparametric model based on the reciprocity and status quo axioms is applied to data from several types of games with reciprocal motivations, some distinguished by acts of commission or omission. 


\section{References}

Andreoni, James and John Miller, "Giving According to GARP: An Experimental Test of the Consistency of Preferences for Altruism,” Econometrica, v. 70, no.2, 2002, pp. 737-753.

Bolton, Gary E. and Axel Ockenfels, "ERC: A Theory of Equity, Reciprocity, and Competition,” American Economic Review, 90, 2000, pp. 166-93.

Charness, Gary and Matthew Rabin, "Understanding Social Preferences with Simple Tests," Quarterly Journal of Economics, 117, 2002, pp. 817-69.

Deck, Cary A., "A Test of Behavioral and Game Theoretic Models of Play in Exchange and Insurance Environments,” American Economic Review, 91, 2001, pp. 1546-55.

Engelmann, Dirk and Martin Strobel, "Inequality Aversion, Efficiency, and Maximin Preferences in Simple Distribution Experiments," American Economic Review, September 94, 2004, pp. 85769.

Fehr, Ernst and Klaus M. Schmidt, "A Theory of Fairness, Competition, and Cooperation,” Quarterly Journal of Economics, August 1999, pp. 817-68.

Güth, Werner, Nadège Marchand, and Jean-Louis Rulliere, "On the Reliability of Reciprocal Fairness-An Experimental Study,” Discussion Paper, Humboldt University of Berlin, 1997.

Hoffman, E., McCabe, K., Shachat, K., and Smith, V. (1994). "Preferences, Property Rights, and Anonymity in Bargaining Games.” Games and Economic Behavior, 7, 346-380.

Roth, Alvin E., Vesna Prasnikar, Masahiro Okuno-Fujiwara, and Shmuel Zamir, "Bargaining and Market Behavior in Jerusalem, Ljublana, Pittsburgh, and Tokyo," American Economic Review, 81, 1991, pp. 1068-1095. 
Table 1. Feasible Choices in Experiment 2

\begin{tabular}{|l|l|l|l|}
\hline$\underline{m}$ & $\underline{y}_{1}$ & $y_{2}$ & $y_{3}$ \\
\hline 15 & 5 & 11 & 11 \\
\hline 15 & 5 & 20 & 20 \\
\hline 15 & 5 & 7 & 38 \\
\hline
\end{tabular}

Table 2. Feasible Choices in Experiment 3

\begin{tabular}{|l|l|l|l|}
\hline$\underline{m}$ & $\underline{y}_{1}$ & $y_{2}$ & $y_{3}$ \\
\hline 15 & 5 & 20 & 20 \\
\hline 15 & 8 & 17 & 20 \\
\hline 15 & 9 & 10 & 26 \\
\hline
\end{tabular}




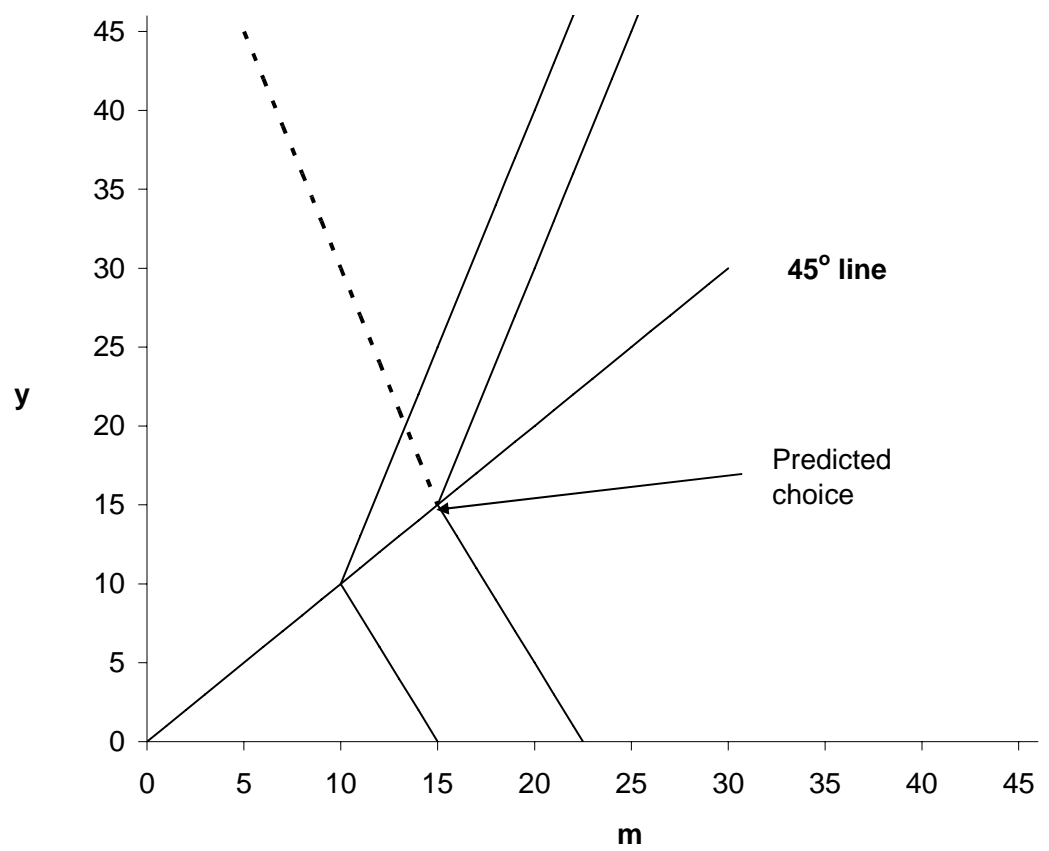

Figure 1. Budget (dashed) Line and Indifference "Curves" for the Fehr- Schmidt Model $(\alpha=1 / 2$ and $\beta=1 / 3)$.

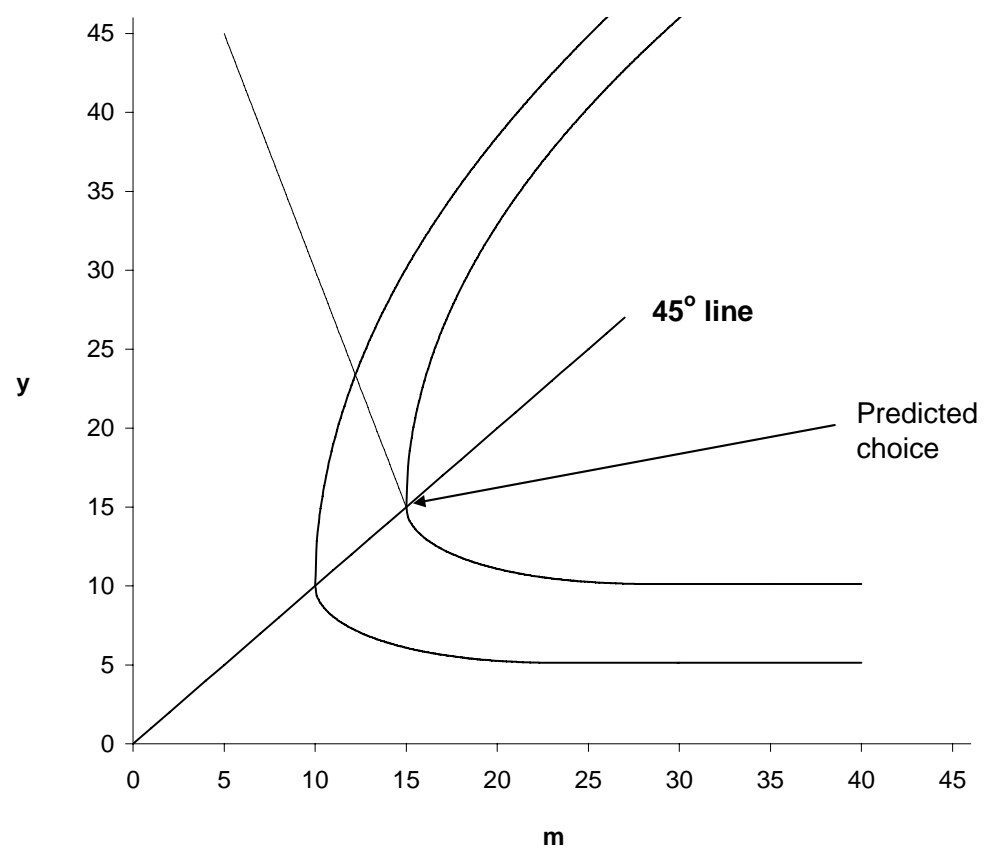

Figure 2. Budget (dashed) Line and Indifference Curves” for the Bolton-Ockenfels Model. 


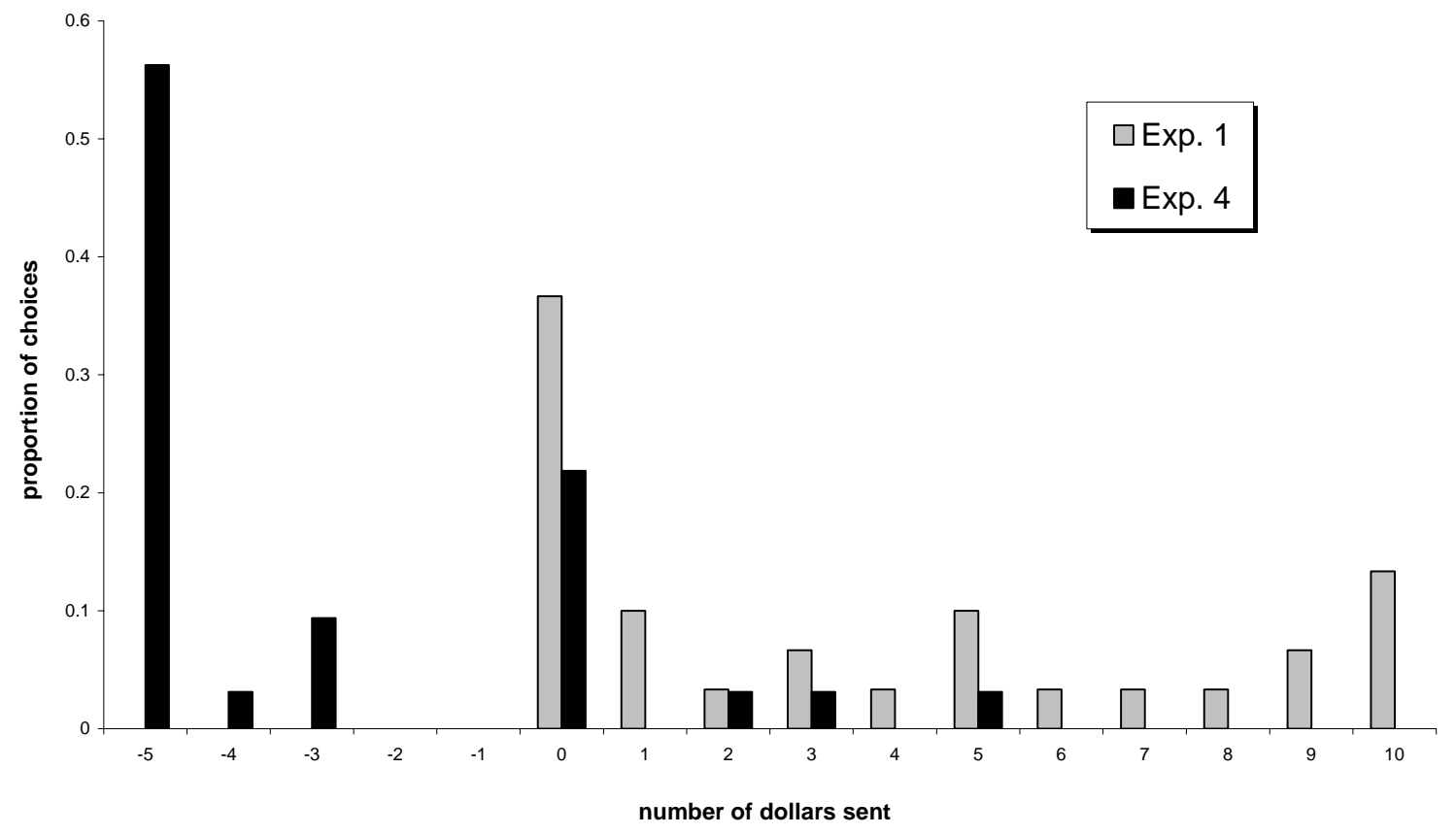

Figure 3. Dictators' Decisions in Experiments 1 and 4

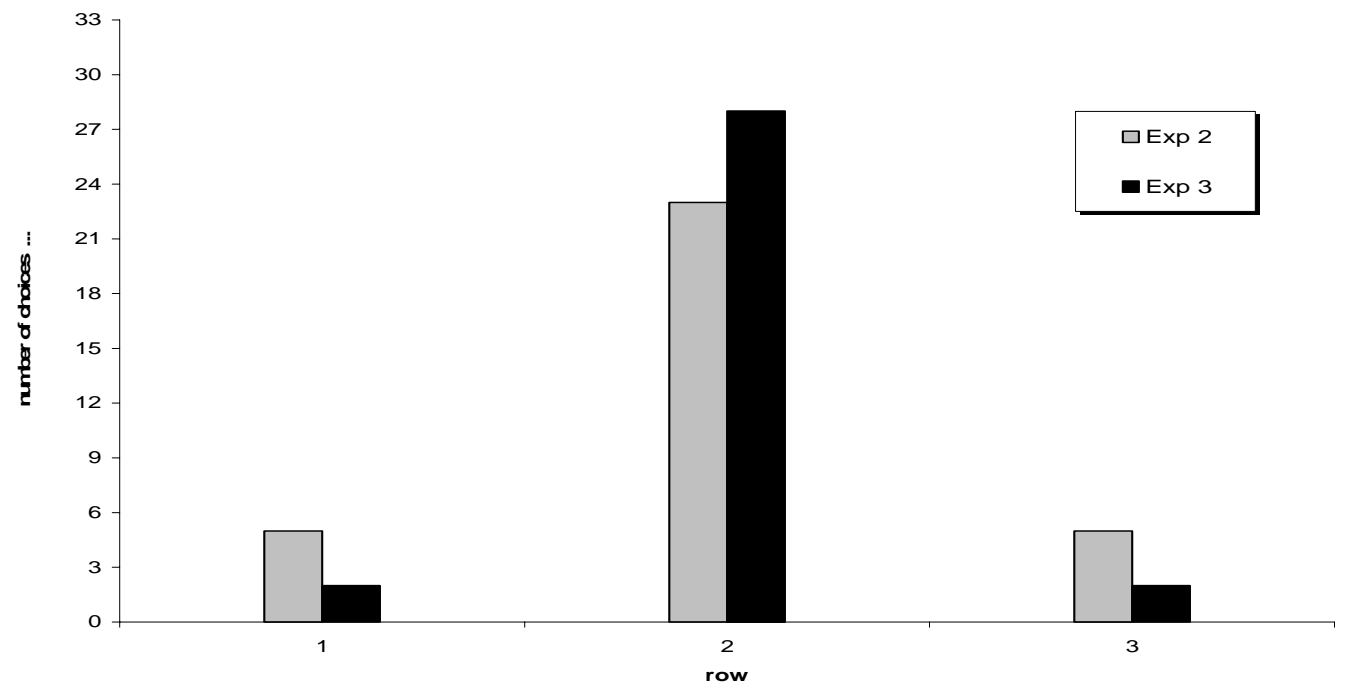

Figure 4. Dictators' Decisions in Experiments 2 and 3 


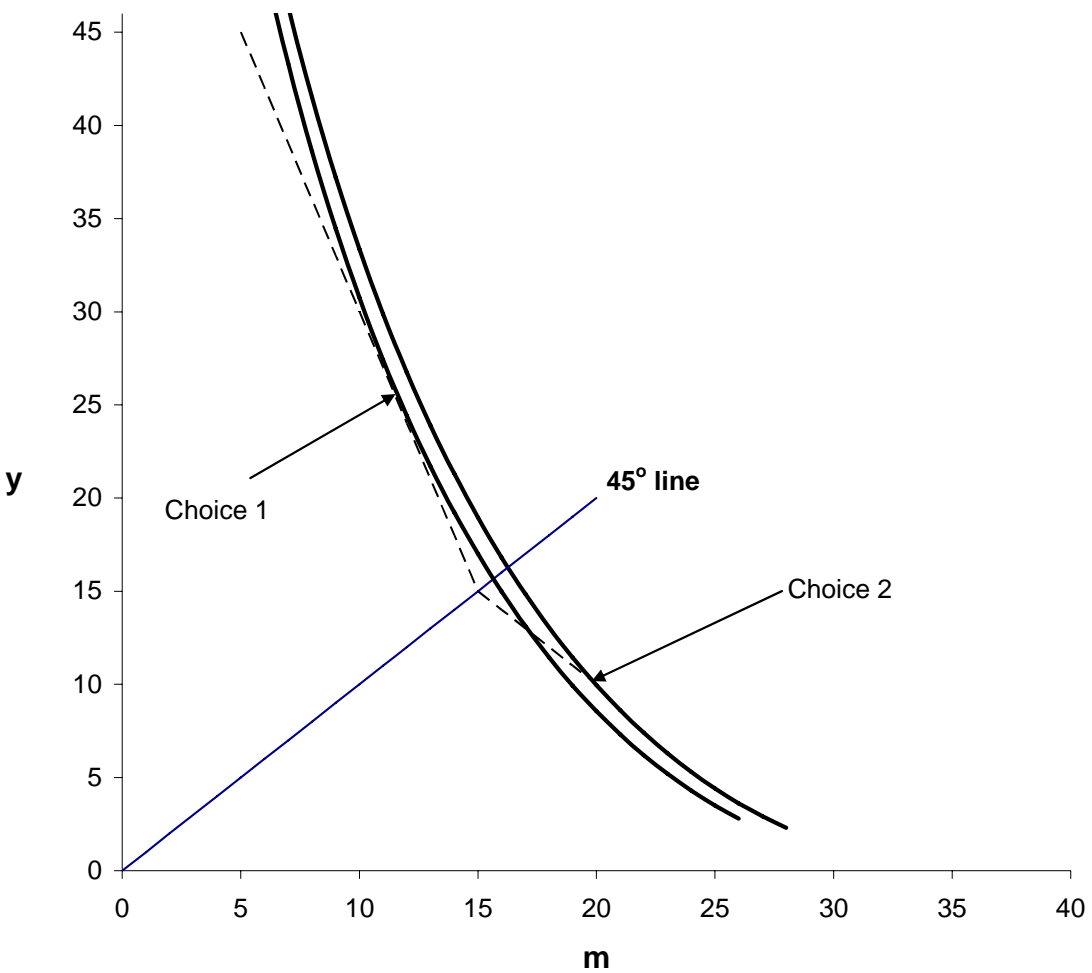

Figure 5. Rationalizing Observations for Experiments 1 and 4 ( $\alpha=0.5$ and $\theta=0.5$ ) 


\section{Appendix 1: Experiment Procedures}

\section{Procedures of Experiment 1}

Experiment 1 was a manual (i.e., non-computerized) experiment with a double-blind payoff protocol. During the decision-making part of an experiment session, all interactions with the subjects were carried out by a "monitor" that had been randomly selected from the subject pool. In addition to distributing and collecting "decision envelopes," the monitor was given the responsibility of verifying that the experimenters accurately implemented subjects' decisions in calculating payoffs. The monitor did not discuss the decision task with the subjects. The monitor was paid \$20. The amount paid to a monitor was not announced to the subjects so as to avoid the possible suggestion of a focal point earnings figure.

The subjects first gathered in a room adjacent to the laboratory. The monitor was randomly selected from the group of subjects by drawing a ball from a bingo cage in the presence of all of the subjects. Next, all of the rest of the subjects were randomly assigned to "group X" and "group Y.” All subjects then entered the laboratory. Group X subjects were seated at widely separated computer terminals with privacy side and front partitions. (The computers were not used.) The group Y subjects were standing at the back of the room. Each subject and the monitor were given copies of the instructions. Then the experimenter read aloud the instructions. After the reading of instructions was completed, the group Y subjects were escorted back to the adjacent room. The group $\mathrm{X}$ subjects had no further contact with the group $\mathrm{Y}$ subjects. Then the group $\mathrm{X}$ subjects were given the opportunity to raise their hands if they had questions. If a subject raised his hand, he was approached by the experimenter and given an opportunity to ask questions and receive answers in a low voice that could not be overheard by other subjects. When there were no more questions, the experimenter left the room and the monitor took over. There was no interaction between the experimenter and the subjects during the decision-making part of an experiment session. All distribution and collection of envelopes containing subject response 
forms was done by the monitor. Because subject decision forms were inside envelopes when they were distributed and collected, not even the monitor could know any subject's personal decision.

The payoff procedure was double blind: (a) subject responses were identified only by letters that were private information of the subjects; and (b) money payoffs were collected in private from sealed envelopes contained in lettered mailboxes. Double blind payoffs were implemented by having each subject draw an unmarked sealed envelope containing a distinctlylettered key from a box containing many envelopes. The subjects wrote their key letters on their response forms; thus payoffs could be correctly made. At the end of the experiment, the subjects used their keys to open lettered mailboxes that contained their money payoffs in sealed envelopes. The experimenters were not present in the mailbox room when the subjects collected their payoff envelopes.

All of the above-described features of the experimental design and procedures were common information given to the subjects before they made their decisions. The subject instructions and response forms did not use evocative labels in referring to the two groups of subjects. Instead, the terms "group X” and "group Y” were used. No subject participated in more than one experiment session.

All of the experiment sessions ended with each subject being asked to earn his $\$ 5$ showup fee by filling out a questionnaire. Group X and group Y subjects had distinct questionnaires. The questions asked had three functions: (a) to provide additional data; (b) to provide a check for possible subject confusion about the decision tasks; and (c) to provide checks for possible recording errors by the experimenters and counting errors by the subjects. Subjects did not write their names or any other identity-revealing information on the questionnaires. The additional data provided by the questionnaires included the subjects' reports of their payoff key letters. Data error checks provided by the questionnaires came from asking the group $\mathrm{X}$ subjects to report the numbers of tokens sent. These reports, together with two distinct records kept by the experimenters, provided accuracy checks on data recording. 


\section{Procedures of Experiment 2}

Experiment 2 was a manual (i.e., non-computerized) experiment with a double-blind payoff protocol. Double-blind payoffs could be, and were, implemented in this experiment without the use of a monitor and mailbox payoff procedure because the payoffs to the dictators were independent of their choices.

The subjects first gathered in a room adjacent to the laboratory. The stations in the laboratory were randomly assigned, in equal numbers, to four groups. This was done independently for each experiment session. A large manila envelope containing experiment documents was placed at each station. Subjects entered the laboratory and sat at any station they chose but without any way of knowing which of the four groups that station had been randomly assigned to. Each station had privacy side and front partitions. Procedural instructions were projected on a screen at the front of the room. The dictators were designated group $\mathrm{W}$. The other subjects were designated groups $\mathrm{X}, \mathrm{Y}$ and $\mathrm{Z}$. The subjects in groups $\mathrm{X}, \mathrm{Y}$, and $\mathrm{Z}$ were given questionnaires to fill out; in this way it was not clear to other groups during the experiment which of the seats were occupied by individuals randomly assigned to be dictators. After the passage of more than enough time for decisions to be recorded, an experimenter asked from a back laboratory door for everyone who had completed his questionnaire to raise his or her hand. After all hands were raised, two messages were alternated on the projection screen at the front of the laboratory. One message instructed group X, Y and Z subjects to "wait for further instructions." The other message instructed group $\mathrm{W}$ subjects to put all of the experiment material except the disclaimer form and the sealed white legal-size envelope back in the large manila envelope and deposit the manila envelope in a box at the front of the laboratory while exiting. They were instructed that the sealed white envelope contained their payoffs ( $\$ 10$ plus the show-up fee of \$5). These envelopes were sealed and had labels attached on both sides with the instruction "not to open this envelope until after exiting the building." After all group W subjects had exited, an 
experimenter retrieved the box and took it to the separate rear "monitor" room. The forms with the group W subjects' decisions were extracted. Then the group X, Y and Z subjects were called, one at a time to receive their show-up fees and the payoffs determined by the group W subjects' decisions. This process involved another randomization: the group $\mathrm{W}$ subjects' decision forms were applied in random order to determine the payoffs of group $\mathrm{X}$, and $\mathrm{Y}$, and $\mathrm{Z}$ subjects. The three screens of projected instructions and the printed instructions contained in the large manila envelopes are available on an experimenter's homepage, as explained in footnote 1.

All of the above-described features of the experimental design and procedures were common information given to the subjects. The subject instructions and response forms did not use evocative labels, such as "dictator" in referring to the four groups of subjects. Instead, the terms "group W," "group X," "group Y” and "group Z” were used. No subject participated in more than one experiment session.

\section{Procedures of Experiment 3}

The experiment procedures and subject instructions for experiment 3 were the same as those for experiment 2 except for the use of the payoffs in Table 2 rather than Table 1.

\section{Procedures of Experiment 4}

The experiment procedures and subject instructions for experiment 4 were the same as those for experiment 1 except for minimal changes necessary to introduce the opportunity for group $\mathrm{X}$ subjects to take money from paired group Y subjects as well as give them money.

\section{Appendix 2: Derivations of Indifference Curves}

\section{Indifference Curves for the Fehr-Schmidt Model}

The F\&S model is based on the assumption that agent $i$, where $i=1,2, \cdots n$, has preferences that can be represented by utility functions of the form 
(A. 1$) \quad u_{i}(x)=x_{i}-\alpha_{i} \frac{1}{n-1} \sum_{j \neq i} \max \left(x_{j}-x_{i}, 0\right)-\beta_{i} \frac{1}{n-1} \sum_{j \neq i} \max \left(x_{i}-x_{j}, 0\right)$

where $\beta_{i} \leq \alpha_{i}$ and $0 \leq \beta_{i}<1$. The utility function for the two-agent case can be written as

(A. 2) $u_{m}(m, y)=m-\alpha(y-m)$, if $m<y$

$$
=m-\beta(m-y) \text {, if } m \geq y
$$

where $m=x_{i}$ is "my income" and $y=x_{j}$ is "your income."

All parameter values that are consistent with inequality aversion imply that the indifference "curves" have positive slope above the 45-degree line because $\frac{d y}{d m}=-\frac{\partial u_{m} / \partial m}{\partial u_{m} / \partial y}=\frac{1+\alpha}{\alpha}>0$ for $m<y$. Figure 1 shows indifference "curves” for the F\&S model for $\alpha=1 / 2$ and $\beta=1 / 3$.

\section{Indifference Curves for the Bolton-Ockenfels Model}

The B\&O model is based on a "motivation function" of the form,

(A. 3) $v_{i}=v_{i}\left(x_{i}, \lambda_{i}\right)$

where

$$
\begin{aligned}
& \text { (A. 4) } \lambda_{i}=x_{i} / \sum_{j=1}^{n} x_{j} \text {, if } \sum_{j=1}^{n} x_{j}>0 \\
& =1 / n \text {, if } \sum_{j=1}^{n} x_{j}=0 \text {. }
\end{aligned}
$$

Given that the sum of my income $(m)$ and your income $(y)$ is positive, the motivation function for the two-agent case can be written as

(A. 5) $v=v(m, m /(m+y))$, 
where $v(\cdot)$ is (B\&O, pgs. 171-172) globally non-decreasing and concave in my income $m$, strictly concave in relative income $m /(m+y)$, and has a partial derivative with respect to relative income with the property

(A. 6) $v_{2}(m, 1 / 2)=0$, for all $m$.

Statement (A.5) and concavity imply that $v_{2}(m, m /(m+y))>0$ for $m /(m+y)<1 / 2$ which is equivalent to $m<y$. Hence, above the 45-degree line indifference curves have positive slope since $\frac{d y}{d m}=-\frac{\partial v / \partial m}{\partial v / \partial y}=\frac{v_{1}(m+y)^{2}}{v_{2} m}+\frac{y}{m}>0$ for such $m<y$. Figure 2 shows typical graphs of the level sets or indifference curves of the B\&O motivation function for the two-agent case with $m+y>0$.

\section{Appendix 3: Derivations of Propositions}

\section{Dictator Game Experiments 1 and 4.}

Denote $f(t)=t^{\alpha}$, if $\alpha \in(-\infty, 1) \backslash\{0\}$ and $f(t)=\ln (t)$, if $\alpha=0$. Then define the following

functions $v:(-\infty, 1)->(1 / 3, \infty)$ such that $v(\alpha)=\frac{f(15)-f(14)}{f(18)-f(15)}$, and $w:(-\infty, 1)->(0,1)$ such that $w(\alpha)=\frac{f(16)-f(15)}{f(15)-f(14)}$

I. $\quad$ Let $s^{a}$ be the amount sent by a dictator in experiment 1 . Then $s^{a} \geq 1$ for all $(\alpha, \theta)$ such that $v(\alpha)<\theta$ and $s^{a}=0$ if $v(\alpha)>\theta$.

II. Let $s^{b}$ be the amount sent by a dictator in experiment 4 . There exists a function $h:(-\infty, 1)->(1 / 3,1)$ such that for any given $\alpha$

a. if $\theta>h(\alpha)$ then $s^{b} \geq 1$ if $v(\alpha)<\theta$ and $s^{b}=0$ if $v(\alpha)>\theta$.

$b$. if $\theta<h(\alpha)$ then $s^{b} \leq-1$ if $w(\alpha)<\theta$ and $s^{b}=0$ if $w(\alpha)>\theta$.

In these dictator games, each dictator and non-dictator is given $\$ 15$. In experiment 1 , a dictator can give the paired person a whole dollar amount in [0,10], whereas in experiment 4 he can give or take away from the paired person a whole dollar amount in $[-5,10]$. Thus, the budget set in experiment 1 is given by $\Omega^{a}=\{(15-s, 15+3 s) \mid s \in[0,10]\}$, whereas in experiment 4 the 
budget set is $\Omega^{b}=\Omega^{a} \cup \Omega^{-}$, where $\Omega^{-}=\{(15-s, 15+s) \mid s \in[-5,0]\}$. The dictator's utility as a function of argument $s$ in experiment 4 is:

$$
\text { (A. } 7) \begin{aligned}
U(s) & =u^{1}(15-s, 15+3 s), \quad s \in[0,10], \\
& =u^{1}(15-s, 15+s), \quad s \in[-5,0) .
\end{aligned}
$$

with $u^{1}(\cdot, \cdot)$ as given by statement (3) in section 4.1 . The dictator's utility as a function of argument $s$ in experiment 1 is given by the first row in (A.7). The slope $d y / d m$ of an indifference curve through a point $P \in \Omega$ in the budget set in experiment 4 is given by

$$
\begin{aligned}
\frac{d y}{d m} & =-\frac{1}{\theta}\left(\frac{15+3 s}{15-s}\right)^{1-\alpha}, \quad \text { if } \quad s \in[0,10], \\
& =-\frac{1}{\theta}\left(\frac{15+s}{15-s}\right)^{1-\alpha}, \quad \text { if } \quad s \in[-5,0],
\end{aligned}
$$

where again the first row is relevant for experiment 1 .

First we derive part I. Note that the slope $d y / d m$ of an indifference curve decreases as we move upward along a budget-line segment, i.e. as $s$ increases. This implies that if the slope of the indifference curve through $(14,18)$ is larger than or equal to the slope of the budget line at $(14,18)$ then the most preferred point for the dictator is $(14,18)$ or some point on the budget line to the left and above it. Thus, if $\frac{d y}{d m}(14,18) \geq-3$, i.e. $\frac{1}{3}\left(\frac{9}{7}\right)^{1-\alpha}<\theta$ then $U(s)$ takes its maximum on $[0,10]$ at

$$
\begin{aligned}
s_{1}(\alpha, \theta) & =15 \frac{(3 \theta)^{1 /(1-\alpha)}-1}{(3 \theta)^{1 /(1-\alpha)}+1}, \quad \text { if } \quad \frac{1}{3}\left(\frac{9}{7}\right)^{1-\alpha}<\theta \leq \frac{5^{1-\alpha}}{3} \\
& =10, \quad \text { if } \quad \frac{5^{1-\alpha}}{3}<\theta<1 .
\end{aligned}
$$


where the first expression is derived by solving $U^{\prime}(s)=0$ for $s>0$ (or $\frac{d y}{d m}(P)=-3$, $\left.\left.P \in \Omega^{a}\right)\right)$. Note that $s_{1} \geq 1$ follows from $\frac{d y}{d m}(14,18) \geq-3$. Let $[x]$ denote the largest integer smaller than $\mathrm{x}$. Then the dictator's most preferred choice $s^{a}$ is to send $\left[\mathrm{s}_{1}\right]$ or $1+\left[\mathrm{s}_{1}\right]$ depending on which is larger, $U\left(\left[s_{1}\right]\right)$ or $U\left(\left[s_{1}\right]+1\right)$. In case that the slope at $(14,18)$ is smaller than the slope of the budget set, which is -3 , then 0 is the most preferred point if and only if $U(0)>U(1)$, which is equivalent to $v(\alpha)>\theta$. This completes the proof of Part I. Note that $s_{1}(\alpha, \theta)$ takes values from 1 to 10 and therefore sending any integer from 1 to 10 which is observed in the data can be explained by this model.

Next we derive part II. Consider the part of the budget set, $\Omega^{-}$in experiment 4 that is below the 45-degree line. Similarly as in experiment 1 , if the slope of the indifference curve through $(16,15)$ (which corresponds to taking away $\$ 1$ ) is smaller than or equal to the slope, -1 of the budget line segment $\Omega^{-}$, then the most preferred choice in $[-5,0]$ is to take away $\$ 1$ or more. In other words, if $\theta \leq(7 / 8)^{1-\alpha}$ then $U(s)$ takes its maximum on $[-5,0]$ at some $S_{2} \in[-5,1]$ given by

$$
\begin{aligned}
s_{2}(\alpha, \theta) & =-15 \frac{1-\theta^{1 /(1-\alpha)}}{1+\theta^{1 /(1-\alpha)}}, \quad \text { if } \quad \frac{1}{2^{1-\alpha}} \leq \theta \leq\left(\frac{7}{8}\right)^{1-\alpha}, \\
& =-5, \quad \text { if } \quad 0 \leq \theta<\frac{1}{2^{1-\alpha}},
\end{aligned}
$$

where the first expression is derived by solving $U^{\prime}(s)=0$ ( or $\frac{d y}{d m}(P), P \in \Omega^{-}$) for $s \in[-5,0]$. Then the most preferred choice, $s_{-}$for the dictator in $[-5,0]$ is to take away $\left[\mathrm{s}_{2}\right]$ or $\left(\left[\mathrm{s}_{2}\right]+1\right)$, depending on which is larger, $U\left(\left[s_{2}\right]\right)$ or $U\left(\left[s_{2}\right]+1\right)$. On the other hand, if the slope of the indifference curve through $(16,14)$ is larger than -1 , which is the slope of the budget-line segment $\Omega^{-}$, then the most preferred choice is either 0 or to take away 1 , whichever has larger 
utility. It can be verified that $U(0)>U(-1)$ if and only if $\theta>w(\alpha)$. Combining these results with the results in Part I, one has the following possible cases. The most preferred choice $s^{b}$ in experiment 4 is:

1. 0 for all $(\alpha, \theta)$ such as $w(\alpha)<\theta<v(\alpha)$. Indeed, from part I $\theta<v(\alpha)$ implies $U(s)<U(0)$ for all whole dollar amounts $s$ in $[0,10]$ and from the last paragraph $w(\alpha)<\theta$ implies $U(s)<U(0)$ for all whole amounts of dollars s in $[-5,0]$.

2. To take away $s_{-}(\leq-1)$ for all $(\alpha, \theta)$ such that $\theta<w(\alpha)$ and $\theta<v(\alpha) . \theta<w(\alpha)$ implies $U(s)<U\left(s_{-}\right)$for all whole amounts of dollars from [-5,0] and $\theta<v(\alpha)$ implies $U(s)<U(0)$ for all whole amounts of dollars $s$ from $[0,10]$. Hence the most preferred choice is $S_{-}$.

3. To give $s^{a}(\geq 1)$ for all $(\alpha, \theta)$ such that $w(\alpha)<\theta$ and $\theta>v(\alpha)$ (similarly as 1 . and 2.).

4. Either take $s_{-}(\leq-1)$ or give $s^{a}(\geq 1)$, whichever has larger utility for all $(\alpha, \theta)$ such that $v(\alpha)<\theta<w(\alpha)$. The following derives regions of $(\alpha, \theta)$ for which take $s_{-}$or give $s^{a}$ is the most preferred choice.

Consider $F:(-\infty, 1) \times(1 / 3,1)->R$ such that $F(\alpha, \theta)=U\left(s_{1}(\alpha, \theta)\right)-U\left(s_{2}(\alpha, \theta)\right)$ and let a function $h:(-\infty, 1)->(1 / 3,1)$ be constructed as follows. For any given $\alpha$, let $h(\alpha)$ denote the value of $\theta$ that solves equation $F(\alpha, \theta)=0$. Such a $\theta$ is unique for any given $\alpha$ since $F(\alpha, \theta)=0$ has a unique solution on $(1 / 3,1)$. This follows from $F(\alpha, \theta)$ and

(i) $\quad \lim _{\theta \downarrow 1 / 3} F(\alpha, \theta)=U(0)-U\left(s_{2}(\alpha, 1 / 3)\right)<0$,

(ii) $\quad \lim _{\theta \uparrow 1} F(\alpha, \theta)=U\left(s_{1}(\alpha, 1)\right)-U(0)>0$,

and

$$
\frac{\partial F(\alpha, \theta)}{\partial \theta}=\frac{\partial U\left(s_{1}\right)}{\partial s_{1}} \frac{\partial s_{1}}{\partial \theta}+\frac{\partial U\left(s_{1}\right)}{\partial \theta}-\frac{\partial U\left(s_{2}\right)}{\partial s_{2}} \frac{\partial s_{2}}{\partial \theta}-\frac{\partial U\left(s_{2}\right)}{\partial \theta}
$$




$$
=\frac{\left(15+3 s_{1}\right)^{\alpha}-\left(15+s_{2}\right)^{\alpha}}{\alpha}>0 .
$$

Statements (i) and (ii) imply that, for all values of $(\alpha, \theta)$ such that $\theta<h(\alpha)$, the dictator's optimal choice is to take $s_{2}$ from the other person whereas for all values of $(\alpha, \theta)$ such that $\theta>h(\alpha)$ the dictator's optimal choice is to give $s_{1}$ to other person. (Of course, the dictator is indifferent between choosing $s_{1}$ or $s_{2}$ for all values of $(\alpha, \theta)$ such that $\theta=h(\alpha)$.) The last result and statements $1-4$ above imply statements (a) and (b) in Part II of Proposition 0.

\section{Proposition 1. Games with Proposer Competition}

In the Roth, et al. (1991) study subjects were paid only for one randomly chosen round. Therefore the payoff vector of $n-1$ proposers and 1 responder before payoff of a salient decision is $x=\left(x_{0}, \ldots, x_{0}\right)$, where $x_{0}$ is the show up fee. Let the maximum offer be $\bar{s} \geq 0$.

Part (1). Suppose that the responder rejects it. Then all players get only the show-up fee $x_{0}$ and therefore, from the monotonicity assumption, the responder is better off by deviating and accepting $\bar{S}$ since then for at least one player the monetary payoff becomes strictly larger than $x_{0}$. Hence, it is a dominant strategy for the responder to accept any offer $\bar{s} \geq 0$.

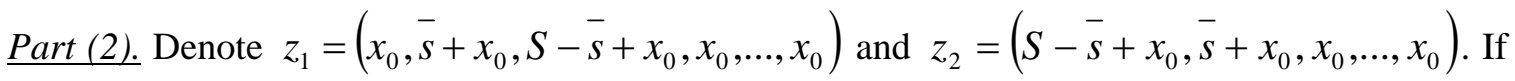
$\bar{S}<S$ then the egocentricity property implies

(A. 11) $u^{1}\left(z_{2}\right)>u^{1}\left(z_{1}\right)$.

a) Suppose that all proposers offer $s=\bar{s}=S-S_{*}$ and the responder accepts it. This is an equilibrium since the responder cannot do better by rejecting, as part (1) shows, and no 
proposer can do better by deviating up or down as follows. The payoff vector for a proposer is $z_{1}$ or $z_{2}$, with probability $1-1 / k$ or $1 / k$. This implies that the expected utility is $E u^{i}\left(S-S_{*}\right)=(1 / k) u^{1}\left(z_{2}\right)+(1-1 / k) u^{1}\left(z_{1}\right)$. Deviating up can only be done by offering $S$, which results in the payoff vector $z^{+}=\left(x_{0}, S+x_{0}, x_{0}, \ldots, x_{0}\right)$. From the convexity assumption, $u^{1}\left(z_{1}\right) \geq u^{1}\left(z^{+}\right)$, which together with (A.11) implies $E u^{i}\left(S-S_{*}\right)>u^{1}\left(z^{+}\right)$. Thus deviating up makes the proposer worse off. Deviating down doesn't make the proposer better off either since it results in the payoff vector $Z_{1}$ for sure and then from (A.11) $E u^{i}\left(S-S_{*}\right)>u^{1}\left(z_{1}\right)$

b) We show that any vector of offers with at least two proposers offering $S$ is equilibrium. In this case the payoff vector for any proposer $i$ is $z^{+}$. Any deviation up or down of a proposer with lower offer than S has no effect on the payoff vector. Similarly, any deviation down by one of the proposers with offer S does not affect the payoff vector either. Therefore no player is strictly better off by deviating from his offer.

c) Let an asymmetric offers vector $\mathrm{O}$ with the highest offer $\bar{S}$ less than $\mathrm{S}$ be given. The payoff vector for a proposer with offer $s$ less than $\bar{s}$ (such a proposer exists because $\mathrm{O}$ is asymmetric) is $z_{1}$. Deviating up and offering $\bar{s}$ makes this proposer better of since the payoff vector in that case is $Z_{1}$ with probability $1-1 / k$ and $z_{2}$ with probability $1 / k$, and (A.11) implies $E u^{i}(S-\bar{s})>u^{i}(s)$.

\section{Proposition 2. Games with Responder Competition}

Let $S^{P}$ be the proposer's proposal in a subgame perfect equilibrium. Part (1). Similar to part (1) of Proposition 1. 
Part (2). Since any offer will be accepted in equilibrium, the proposer's offer is determined by $s^{P}=\arg \max \{E U(s) \mid s \in[0, S]\}$, where $E U(s)$ is defined in statement (10) in section 5.3.2. Differentiating (10) with respect to $s$, one has

$$
\begin{aligned}
\frac{d E U(s)}{d s} & =-\left(x_{1}+S-s\right)^{\alpha-1}+\theta(y+s)^{\alpha-1}, \quad \text { if } \quad \alpha \in(-\infty, 1) \backslash\{0\}, \\
& =(\mathrm{s}+\mathrm{y})^{\theta-1} \mathrm{y}^{(\mathrm{n}-2) \theta}\left(\left(\mathrm{S}-\mathrm{s}+\mathrm{x}_{1}\right) \theta-\mathrm{s}-\mathrm{y}\right) \quad \text { if } \quad \alpha=0 .
\end{aligned}
$$

Let $s^{*}$ denote the value of $s$ that solves equation $d E U(s) / d s=0$, i.e. $s^{*}=\frac{\left(x_{1}+S\right) \rho-y}{1+\rho}$ where $\rho=\theta^{1 /(1-\alpha)}$. We conclude that

$$
S^{P}=0, \quad \text { if } \rho<y /\left(x_{1}+S\right) \text {, }
$$

(A. 13) $=S$, if $\rho>(y+S) / x_{1}$, $=s^{*}$, otherwise. 